\title{
DESARROLLO E IMPLANTACIÓN DE UNA PLATAFORMA WEB PARA LA COMUNICACIÓN INTERNA Y CON LAS FAMILIAS UTILIZANDO G SUITE
}

\author{
DEVELOPMENT AND IMPLEMENTATION OF A WEB PLATFORM \\ FOR BOTH INTERNAL COMMUNICATION AND COMMUNICATION \\ WITH FAMILIES USING G SUITE
}

\section{José Manuel Poveda-Moragón ${ }^{a^{*}}$ y Nestor Martínez-Ballester ${ }^{a}$}

Fechas de recepción y aceptación: 23 de julio de 2019, 3 de diciembre de 2019

Resumen: En un centro escolar se genera constantemente información, por lo que la buena gestión que se hace de ella es muy importante para una buena coordinación. Tanto los trabajadores como las familias tienen derecho a acceder a determinada información que ayude a mejorar el proceso enseñanza-aprendizaje de sus hijos e hijas para unos, alumnos y alumnas para otros, y que, además, pueda hacerlos mejores personas. Gracias a Google y las herramientas de explotación que ofrece de manera gratuita a los centros escolares se ha podido crear una infraestructura tecnológica propia, compuesta de diferentes plataformas web también propias y creadas por el profesorado de nuestro centro e impulsada desde el equipo directivo. Para ello, se han utilizando los recursos de software gratuitos que ofrece Google y que hacen posible un acceso a la información rápido y fiable por parte de las familias y personal del centro educativo. Los beneficios que la creación de esta infraestructura tecnológica supondrá para la comunidad educativa (familias, alumnado, profesorado, PAS y equipo directivo) son muchos, pudiéndose medir sus resultados con indicadores tanto de rendimiento como de satisfacción y aplicaciones gratuitas de análisis de datos.

Palabras clave: información, Google para educación, infraestructura tecnológica, plataforma web, software gratuito, enseñanza, intranet, G Suite.

\footnotetext{
${ }^{a}$ Docentes del Colegio La Purísima. Fundación Educativa Franciscanas de la Inmaculada.

* Correspondencia: Colegio La Purísima. Avenida Tres Cruces 49. 46018 Valencia. España.

E-mail: josemanuelpoveda@lapurisimavalencia.com
} 
Abstract: In a school, information is constantly generated, which means that the adequate management of it is very important for good coordination. Both workers and families are entitled to access certain information that helps improve the teaching-learning process of pupils, and that can also make them better people. Thanks to Google and its free exploitation tools for schools, it has been possible for our school to create our own technological infrastructure, which is made up of different web platforms. It has been created by some of the teachers who work at school and promoted by the school board team. In order to design it, teachers have used Google's free software resources. Thanks to it, now it is possible for families and staff of our school to access information quickly and reliably. The whole educational community (that is to say, families, students, teachers, PAS and school board team) will benefit from the creation of this technological infrastructure whose results will be measured using both performance and satisfaction indicators as well as free data analysis applications.

Keywords: information, Google for education, technological infrastructure, web platform, free software, teaching, intranet, G Suite.

\section{INTRODUCCIÓN}

"Ya no estamos en la era de la información. Estamos en la era de la gestión de la información" Comein (2014)

En un centro escolar se generan miles de datos nuevos que están constantemente actualizándose, por ello esta frase de Comein es muy ilustrativa en nuestro caso. Las personas que gestionan los datos deben tener claro qué información se debe trasladar o recabar; por ello se hace necesaria la creación de una plataforma web/online que sea capaz de canalizar toda la información en el sentido correcto. Dado que la información que necesitan y generan tanto el profesorado como las familias no es la misma, es necesario crear dos tipos de acceso a la plataforma, uno exclusivo para el profesorado, en el que se maneja información de tipo organizativo relacionada con el funcionamiento del colegio, sustituciones, actas de reunión, horarios, etc., e información de tipo educativo, relacionado con los datos obtenidos tanto de las familias como del alumnado, así como un acceso a la plataforma para familias, de uso exclusivo para padres y madres y al cual también puede acceder el profesorado. Es importante saber que el acceso a la información de la plataforma no es abierto y que en función de la relación con el alumnado se otorgarán una serie de permisos para poder visualizar o no una determinada información. 
Es muy importante destacar que la creación de esta plataforma web no va a suponer coste alguno, ni para las familias ni para el colegio, al usarse las infraestructuras y los recursos humanos que ya se tienen y que permiten el acceso a la red de manera rápida y segura. Las familias, para poder acceder a la información, solo necesitan un dispositivo electrónico con conexión a internet, como puede ser un teléfono inteligente. Por otro lado, se utilizarán las herramientas de G Suite que Google ofrece gratuitamente a los colegios y que permitirán hacer y organizar la plataforma web.

En la implantación de la plataforma, es clave el respaldo y patrocinio del equipo directivo del colegio en su estrategia de agilizar la comunicación entre las personas que conforman la comunidad educativa, teniendo claro que este proyecto, no es solo un proyecto TIC, ya que las TIC únicamente darán el soporte y las herramientas tecnológicas necesarias para poder implantar y desarrollar la plataforma web, y sí un proyecto en el que se trata de poner al alcance de manera segura y rápida la información que se genera para los que la necesitan.

\section{MARCO TEÓRICO}

\subsection{El valor estratégico de la comunicación interna/externa corporativa}

Tomando como punto de partida la definición de colegio que da el reglamento de régimen interno de nuestro centro “...una Comunidad Educativa integrada por el conjunto de personas que, relacionadas entre sí e implicadas en la acción educativa, comparten y enriquecen los objetivos del Centro" (Purísima-Franciscanas, 2015), se entiende que una manera de poder implicar a todos los estamentos de una comunidad educativa para asumir una manera de educar y poder ir en la misma dirección es compartir la información.

\subsection{La organización interna como clave del buen funcionamiento}

Una de las claves del buen funcionamiento de cualquier organización, y un colegio lo es, será la existencia de un sistema de comunicación, que como 
afirma Soria (2008) debe estar basado en un plan estratégico. Todo colegio ha de tener una organización interna que permita que este pueda funcionar de manera coordinada y coherente, en la que cada uno de sus miembros forma parte de un organigrama, y cuyas partes, por muy alejadas que estén jerárquicamente, puedan estar relacionadas. Tanto las personas, individualmente, como la empresa, en este caso un colegio, deben organizarse y, por supuesto, agruparse constantemente, a pesar de que siempre existirán limitaciones (Soria, 2008). Como dice González (2015), todo el conocimiento que genera una empresa (colegio) se ve como un activo organizacional, dependiendo su valor de la forma en el que se aprovecha para obtener una ventaja o conseguir una meta. Esa gestión del conocimiento, atendiendo a lo que afirma González, sigue siendo una frontera en la actividad de una organización.

Está demostrado que una buena comunicación puede evitar conflictos y mejora la satisfacción del trabajador (Casares, 2007) y, por extensión, de las familias, entre otros efectos, porque de esta manera, como afirma Santana (2013), "podemos transmitir correctamente a los empleados los objetivos y valores estratégicos que promueve la organización y generar una cultura de apropiación, fidelidad y sentido de pertenencia”. En la misma línea, Santana afirma que ese sentido de pertenencia mantiene integrados a los trabajadores y alineados con la filosofía de la organización, con la circulación de mensajes que inculquen la misión, visión, valores, estrategias y metas corporativas; en el caso de un colegio, su misión y visión son las que fundamentan su carácter propio.

Siguiendo con el estudio hecho por Soria (2008), la mejora del proceso de comunicación aumenta la transferencia de información entre las personas de una organización, pudiendo compartir ideas y opiniones, lo que mejora la realización de actividades que son necesarias para el funcionamiento de una organización. En un centro escolar, el número de actividades que se realizan a lo largo de un curso es muy elevado, y aunque estas se dan en grupos de edades muy dispares, deben estar vertebradas para alcanzar unas mismas metas. De la misma manera, son varios los profesionales que deben acceder a la información personal del alumnado, y el no poder acceder o recibir la información con rapidez puede limitar las acciones de atención que necesitan u ocasionar que realicen muchas menos. Por ello y haciendo un estudio previo en el que se puedan analizar todas las necesidades de comunicación que tiene el equipo 
docente, no docente y las familias de un colegio, se podrá elaborar un plan de comunicación en el que todos puedan acceder a la información de una fuente fiable de una manera rápida y segura.

\subsection{Organización de la comunicación en una organización}

La comunicación organizacional es, o debe ser, un conjunto de técnicas y actividades que facilitan el flujo de los mensajes que se dan entre todos los miembros de la organización o entre esta y su entorno (Fernández, 1997). Fernández sigue afirmando que el flujo de mensajes influye en las aptitudes y conductas de los miembros de la asociación con la finalidad de que esta cumpla mejor y más rápido sus objetivos (Fernández, 1997). Por otro lado, Nosnik (1996) indica los cinco rasgos que ha de tener una comunicación para que sea efectiva: abierta, para la comunicación exterior. Hace alusión al medio más usado por la organización para enviar mensajes tanto al exterior como al interior. Con esto se consigue evitar lo que antes, donde la información obedecía a un flujo jerárquico (González, 2015), ahora permite compartir conocimientos dentro y fuera de la organización, pudiendo ampliar los conocimientos en distintos campos (Boersma y Sytze, 2011). La comunicación también ha de ser evolutiva, sobre todo por la comunicación imprevista que se origina; flexible, puesto que permite tener una comunicación entre lo informal y lo formal; multidireccional, manejándose la información en todas las direcciones y sentidos, de abajo hacia arriba, de arriba hacia abajo, de manera transversal, y así, como sugiere Santana (2013), se permite entablar un diálogo constante entre colaboradores y superiores, para no pensar que los primeros son únicamente los que reciben órdenes o solo escuchan informaciones. La motivación del talento se potencia con la retroalimentación y evaluación conjunta de las funciones y los indicadores de cumplimiento. Por último, la comunicación, para que sea efectiva, debe ser instrumental, es decir, se deben utilizar herramientas como soportes, pues hay organizaciones que funcionan de manera poco eficiente debido a que la información no llega de una manera rápida, lo que provoca que la comunicación sea poco efectiva. Esta red interna de comunicación que se tiene que crear o intranet es muy recomendable 
por la inmediatez y las posibilidades de interacción, puesto que permite una motivación del talento (Casares, 2007).

De los cinco tipos de trasmisión de la información que según Katz y Kahn (1990) (Katz y Kahn, 1990) existen, se puede observar, a su vez, distintos tipos de comunicación: descendente, ascendente, horizontal, informal y rumor; este último tipo es el que puede dañar la imagen de una organización, entre otras cosas y según dicen los autores, los rumores son comunicados sin fundamento o evidencias formales que se transmiten de forma unipersonal y que utilizan medios informales (Martínez de Velasco, Nosnik, Vargas y Savage, 1988). Un colegio, como organización que es, debe evitar que este tipo de información no comprobada circule entre sus miembros y, como afirma Santana (2013), esto se consigue con una política comunicativa adecuada que informe de lo que está ocurriendo en el interior y que contrarreste los rumores externos, que pueden provocar medios de comunicación o declaraciones interesadas de cariz político.

La comunicación, explica Soria (2008), es un proceso humano que genera vínculos entre humanos e implica una serie de principios que Nosnik (1990) ha denominado "las tres íes" de la comunicación en una organización, que se deben tener muy presentes en un centro educativo: comunicación íntegra o veracidad en todas las comunicaciones que se dan, basadas en hechos verificables; comunicación integral, porque debe cumplir con todos los receptores de una organización; y comunicación integrada, dejando de ser esta materia de especialistas, de manera que se genera una plataforma de información y su manejo, lo que mejora la coordinación.

\subsection{G Suite para centros educativos de Google}

G Suite nos ofrece las siguientes herramientas, todas ellas con posibilidad de compartir con otros usuarios dando permisos de escritura, para comentar únicamente o solo de lectura (MECD, 2015):

- correo gmail, para acceder a las cuentas corporativas creadas por el centro escolar; 
- Google Calendar, donde se pueden generar horarios de clase, programar reuniones de claustros, consejos escolares, equipos docentes... Además, se pueden añadir varios calendarios, donde se apuntan los eventos, a los cuales pueden confirmar su asistencia los usuarios invitados;

- Google Drive, para gestionar diferentes tipos de documentos en la nube; además, permite dar los permisos de visión o edición a los archivos que en ella se almacena;

- Google Docs, Sheets y Slides, que son los documentos propios de Google, entre los que existen diferentes tipos: documentos de texto, hojas de cálculo y presentaciones, donde los documentos y sus modificaciones quedan registradas en la nube; y

- Google Sites, que permite crear sitios web dinámicos y seguros sin necesidad de conocimientos de programación ni HTML. Su visualización se adapta perfectamente al tipo de dispositivo electrónico desde el que se accede.

\subsection{Google Analytics}

Google Analytics es una herramienta gratuita de análisis de sitios web muy completa que nos facilita información como el número de visitantes, qué y en qué orden visitan las diferentes páginas web dentro de la plataforma web, el dispositivo electrónico con el que visitan la página, el rango de edades de los visitantes, etc.

Gracias a Google Analytics se pueden analizar todos los datos relacionados con los usuarios de la plataforma web creada, tanto de profesorado como de familias, para así mejorar diferentes aspectos interesantes y poder ofrecer un servicio cada vez más personalizado y próximo a sus necesidades informativas.

\section{Metodología}

La metodología utilizada será empírica y cualitativa, basada en la investigación-acción que Lomax (1990) define como "una intervención en la práctica 
profesional con la intención de ocasionar una mejora”. Esta intervención comenzará con una investigación. Como describe Latorre (2003), esto se debe llevar a cabo utilizando el triángulo de Lewin (1946), que se apoya en tres pilares: la investigación, la acción y la formación, para el desarrollo de cualquier profesional.

Atendiendo a lo descrito por Lewin (1946) y que recoge Latorre (2003), la metodología de investigación-acción dibuja una espiral de pasos: planificación, puesta en marcha y evaluación del resultado. La investigación-acción tiene un doble propósito: acción para cambiar una institución, e investigación para generar conocimiento. Por lo tanto, la investigación-acción no es ni investigación ni acción, sino la relación de ambas en un bucle infinito.

El plan para la puesta en marcha de la metodología basada en la investigación-acción que se va a realizar en el colegio consta de cuatro fases:

FIGURA 1

Fases en la investigación-acción para la puesta en marcha del proyecto

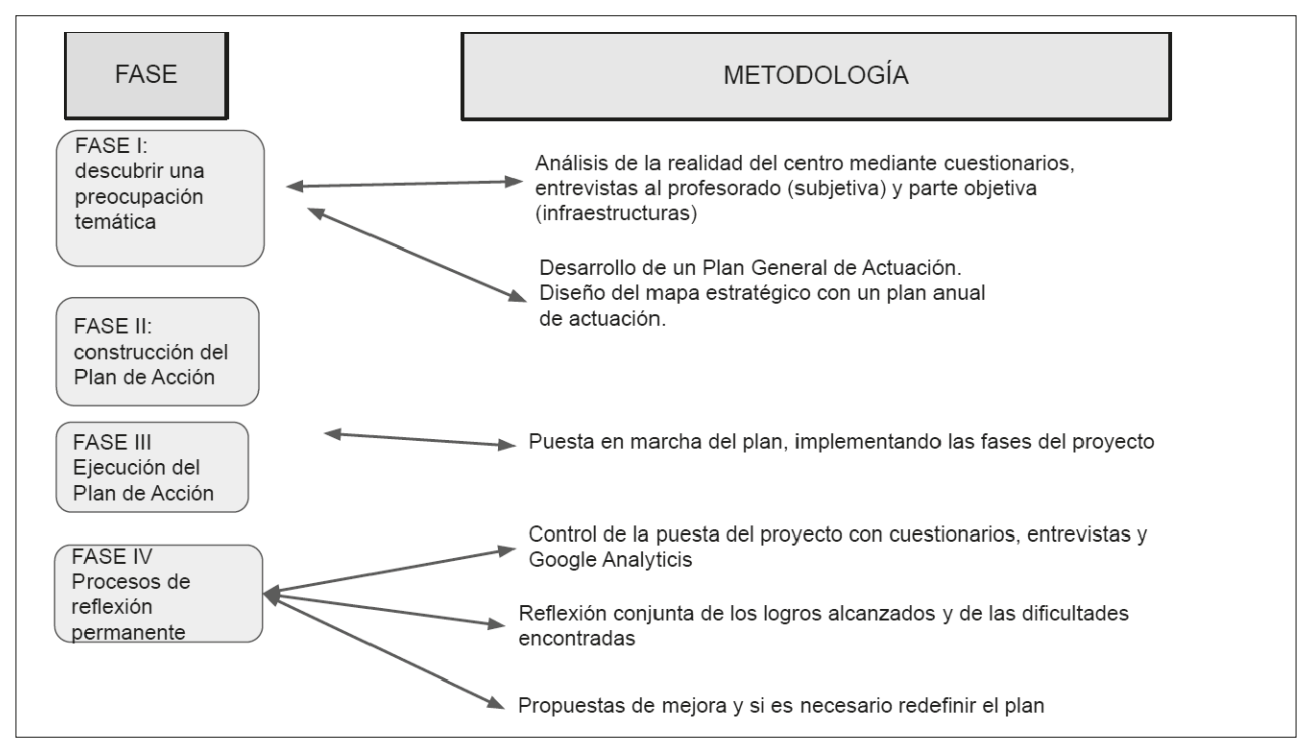

Fuente: elaboración propia 
Atendiendo a Latorre (2008) la institucionalización de este método conllevará unos cambios organizativos y personales, ya que se pondrán en marcha nuevos procesos y estrategias de manera que darán estabilidad y continuidad a los cambios introducidos en la utilización y transmisión de la información.

\section{Desarrollo}

La implantación de la plataforma se realizará en varias fases. En algunos casos el paso a la siguiente fase no se podrá realizar hasta que la anterior no esté finalizada; en otros, podrá comenzar una fase sin haber finalizado la anterior. Lo que no se podrá hacer bajo ningún concepto es forzar la implantación de las fases. La duración que se da más adelante de cada fase se debe tomar como orientativa, pudiendo durar más o menos dependiendo de las características particulares del centro donde se implante la plataforma.

\subsection{Fase 0. Alta en G Suite para centros educativos y verificación del dominio web}

La G Suite para centros educativos ofrece recursos de software de manera gratuita a los centros educativos sin ánimo de lucro. Se tendrán que facilitar una serie de datos que identifiquen al centro como un colegio para poder acceder a todos los servicios que nos ofrece.

Duración: aproximadamente dos semanas.

Recursos humanos: Coordinador TIC.

\subsection{Fase 1. Alta de usuarios y configuración de aplicaciones de G Suite}

Se crearán la cuentas corporativas @lapurisimavalencia.com al profesorado y PAS,@fam.lapurisimavalencia.com a las familias y@alu.lapurisimavalencia.com al alumnado (a partir de primero de secundaria), para que puedan acceder a la plataforma web creada y a todo su contenido, tanto aplicaciones como información. 
Duración: dos semanas.

Recursos humanos: Coordinador TIC.

\subsection{Fase 2. Configuración e implantación G Suite}

Se configura G Suite y otros parámetros como, por ejemplo, los ajustes de dispositivos móviles Android que usen una cuenta corporativa del centro, que están disponibles para centros educativos.

Duración: dos semanas.

Recursos humanos: Coordinador TIC.

\subsection{Fase 3. Detección de las necesidades formativas y formación del profesorado}

El profesorado debe conocer las herramientas de la G Suite con las que se hace la plataforma. A partir de las necesidades individuales, se organiza la formación en grupos de trabajo siempre en horario de trabajo. El objetivo es que se aumente la competencia digital del profesorado con más dificultades, con lo que se pretende evitar que el proyecto tenga un rechazo desde su fase inicial por parte del profesorado más reticente al cambio en el canal de trasmisión de la información, al tener una competencia digital baja.

Duración: un curso escolar; luego se realizarán monográficos de ampliación.

Recursos humanos: coordinador del ciclo, coordinador TIC y profesorado formador voluntario.

\subsection{Fase 4. Elaboración del listado de procesos e instrucciones susceptibles de ser compartidos con las familias y personal del centro a través de la plataforma}

Se elaborará una relación de todos los tipos de información y sus responsables de los datos que se comparten y se reciben por parte del profesorado 
y de las familias. Se elaborará otra relación de información y datos que no se comparten por la falta de una herramienta eficaz y/o segura a la vez que sostenible en el tiempo, que sería muy conveniente que se compartiese.

Duración: un curso escolar.

Recursos humanos: departamento de calidad, equipo directivo.

\subsection{Fase 5. Creación e implantación y consolidación de la plataforma web del profesorado}

Las herramientas que se utilizan para la creación de la plataforma web con acceso del profesorado son principalmente Sites y Drive. Con la primera se puede crear una página web muy vistosa y sin necesidad de tener conocimientos en programación, lo que servirá de soporte para acceder a la información que se quiera compartir. Es conveniente que el diseño de esta web lo realice la persona que va a publicar en ella la información, o que al menos esta persona participe en su diseño. Una vez diseñada, el mantenimiento de la página y la publicación de la información son muy fáciles y requieren muy poco tiempo. La herramienta Sites se complementa con Drive, nube de almacenamiento a la cual se vinculará toda la información que se publique y/o se recoja a través del sitio web.

A continuación se enumeran algunos ejemplos de los más de cincuenta tipos de información y datos que se pueden consultar desde cualquier lugar y a cualquier hora accediendo a la plataforma web del profesorado, con la garantía de que los datos están actualizados en tiempo real. Antes, esa misma información solo era posible transmitirla y/o recibirla buscando a las personas que la gestionaban en grupos o reuniones de trabajo, en actas o en documentación impresas, en grupos de WhastApp, por correo electrónico, etc., lo cual suponía un gran "pérdida" de tiempo de trabajo solo en transmitir o recibir la información.

Ejemplos de información publicados en la plataforma web del profesorado: planilla de sustituciones del profesorado; actas de reunión de grupos de trabajo y documentación de cualquier tipo del alumnado, metodologías de trabajo con cada grupo, etc.; cualquier tipo de listado y/o base de datos con información del alumnado y personal; comunicación de incidencias de 
cualquier tipo, disciplinarias, de mantenimiento, de recursos TIC, etc.; calendarios de varios tipos, como son los de ocupación de espacios, fechas de actividades, de reuniones, de exámenes, etc.; protocolos y manuales de actuación ante diversas situaciones, como puede ser un accidente escolar, o una evacuación de emergencia; instrucciones concretas y comunicados internos; datos y teléfonos de contactos; avisos urgentes; cursos realizados por el profesorado; evaluación de actividades; etc. Es importante hacer constar que al trabajar con documentos colaborativos, la información será actualizada por sus responsables en tiempo real, y esta actualización aparecerá reflejada al instante en la plataforma.

Duración: siempre.

Recursos humanos: gestor de la plataforma y responsables de actualizar cada documento.

\subsection{Fase 7. Creación, implantación del acceso a la Intranet Familias y acompañamiento de estas}

Una de las claves del éxito de la plataforma web de familias es la selección de la información que en ella se publica. Las tutorías personalizadas del profesorado con las familias es una manera directa de conocer el tipo de información que estas demandan al colegio sobre sus hijos e hijas y las actividades y/o metodologías que se dan en él.

A continuación se nombran algunos de los más de treinta tipos de información y/o datos que se comparten con las familias en plataforma web familias y que antes solo se les podía hacer llegar en reuniones informativas, en entrevistas personales por circulares impresas o en la secretaría del colegio.

Ejemplos de información publicada: avisos urgentes o recordatorios; circulares informativas y actas de reuniones generales; incidencias y reconocimientos relacionados directamente con su hijo/a; faltas de asistencia y/o retrasos de su hijo/a; calendarios en los que aparecen fechas de exámenes, entregas de trabajos, actividades, etc.; autorización para la realización de actividades extraescolares; información académica como pueden ser criterios de evaluación y calificación, recuperaciones de pendientes, boletín con calificaciones trimestrales, etc. 


\section{Duración: siempre.}

Recursos humanos: gestor de la plataforma y responsables de actualizar cada documento.

\subsection{Fase 8. Mantenimiento. Personalización. Desarrollo a medida. Mejorar la plataforma web}

En esta fase se va a realizar un mantenimiento de las nuevas plataformas web. Esto conlleva mantenerlas actualizadas con las últimas versiones y realizar cambios para su mejora. También se sigue con la programación a medida de la plataforma web, ampliando sus funcionalidades: scripts con nuevas funcionalidades, app para móvil con notificaciones de faltas de asistencia, etc.

Duración: siempre.

Recursos humanos: coordinador TIC.

\section{ANÁLISIS Y EVALUACIÓN}

5.1 Evaluación de la plataforma por parte del profesorado y de las familias

Para comprobar la utilidad y uso de la plataforma y detectar así sus puntos fuertes y débiles se van a utilizar los siguientes instrumentos de medida: encuestas de satisfacción y entrevistas personales tanto con profesorado como con las familias y análisis de los datos de Google Analytics.

\subsection{Entrevistas personales con profesorado y familias}

Con algunos miembros del claustro de las distintas etapas educativas y del PAS, se mantendrán de manera periódica reuniones formales/informales donde se recojan sus impresiones sobre la plataforma web. Por otra parte, en la entrevistas que mantiene periódicamente el profesorado con las familias se les preguntará expresamente sobre el uso y sugerencias de la plataforma web de familias. 


\subsection{Cuestionario de satisfacción y de uso al personal y a las familias sobre el uso de la plataforma web}

Al profesorado y a las familias, con una periodicidad de dos años, se les pasa un breve formulario en el que se preguntan sobre aspectos generales de la plataforma. En el formulario, la mayor parte de las preguntas son de percepción del efecto que ha tenido y tiene la implantación de la plataforma como canal formal de comunicación, sin hacer preguntas para obtener datos de rendimiento que ya se obtienen con Google Analytics.

Una de las preguntas que se hacen tanto a familias como a profesorado es acerca de lo que piensan de la mejora de la comunicación con la plataforma: "Desde que se ha creado la plataforma web, ¿piensas que ha mejorado la comunicación?", con cuatro posibles respuestas:

- Siempre

- Casi siempre

- Pocas veces

- Nunca

En la encuesta también hay una sección donde tanto las familias como el profesorado pueden hacer las valoraciones, sin límite de palabras, que crean oportunas sobre la plataforma web.

\subsection{Google Analytics}

Esta herramienta proporcionará las métricas de uso de las plataformas, para saber a qué secciones acceden más los usuarios y qué inquietudes tienen y, así, aproximar cada vez más la información publicada según sus necesidades.

Los resultados obtenidos con los anteriores instrumentos de medida antes mencionados para la plataforma web del profesorado son los siguientes:

Se realizan más de 30.000 consultas de información en un curso escolar por parte del profesorado, 60 personas en el caso de nuestro centro, desde 
Infantil a Bachillerato. Este dato numérico adquiere más relevancia cuando se obtienen los resultados de satisfacción del profesorado, de los que destacamos los siguientes: el $100 \%$ considera que ha aumentado la coordinación entre dirección y profesorado; el $97 \%$ considera que el uso de la plataforma ha mejorado su competencia docente en el uso de las TIC; el $90 \%$ piensa que ha aumentado la colaboración entre los compañeros; el $100 \%$ consulta la plataforma web todos los días; el $100 \%$ considera que con la plataforma web puede aprovechar mucho mejor el tiempo de trabajo.

Con respecto a las familias, los indicadores de uso muestran que en el primer año de implantación de la plataforma web, las familias de Secundaria han realizado más de 10.000 consultas, eso sin contar los emails que se han enviado de manera automática desde la plataforma a sus cuentas corporativas, informándoles de algún tipo de incidencia o comunicado.

Por otro lado, al ser un servicio gratuito para las familias, hace que sea todavía mucho mejor aceptado por ellas, valorando muy positivamente la iniciativa de su puesta en marcha por adaptarse a sus necesidades sin necesidad de pagar.

Los resultados más destacables obtenidos de las familias son los siguientes: el $62 \%$ de las veces acceden desde su teléfono inteligente y el resto desde un PC; el $26 \%$ accede todos o casi todos los días, siendo el $37 \%$ el que accede una o dos veces por semana. El resto de familias lo hace cuando tiene que buscar una información concreta; el $97 \%$ califica de excelente/bien que el acceso a la plataforma se haga privadamente, es decir, identificándose con su cuenta corporativa; el $88 \%$ de las familias que responden consideran que con la plataforma ha mejorado siempre o casi siempre la comunicación familia-colegio; el 97 \% piensa que la información que se publica o se le envía relacionada con su hijo/a es útil o muy útil. Otros datos muy interesantes son que el $94 \%$ de las familias consideran que el servicio no sería mejor si se utilizase una plataforma de pago; que todas las secciones son valoradas como útiles o muy útiles por más del $90 \%$ de las familias; que el $41 \%$ de las familias no han necesitado soporte técnico por dificultades de acceso, y que de las que lo han necesitado el $92 \%$ considera que se le ha resuelto satisfactoriamente la duda o la incidencia. 


\section{Conclusiones}

La plataforma web tanto de profesorado como de familias en un centro escolar es necesaria por muchas razones, siendo una de ellas que, una vez puesto en marcha el proyecto, no sería fácil imaginarse cómo se trataría toda la información que se genera en un colegio si la plataforma desapareciese.

Una vez analizados los resultados, se concluye que la aceptación de la plataforma por parte del profesorado y de las familias que la usan es muy alta, estando satisfechos ambos grupos de la información publicada, tanto general como la que necesitan de manera específica.

La plataforma web del profesorado permite el acceso a la información que la dirección quiere que esté a disposición de aquel que la necesite de manera rápida, directa y con seguridad, sin que exista pérdida ni una mala interpretación de los datos, y sin que puedan acceder personas no autorizadas. Todo ello aumenta la coordinación entre el personal y disminuye el tiempo utilizado en acceder a la información, de manera que este tiempo "ganado" se utiliza en mejorar la calidad educativa, al ser mucho más eficientes en el tiempo de trabajo en el caso del profesorado y en el de la gestión en el caso de la dirección. Por otro lado, tener acceso a la información más relevante relacionada con el colegio hace que el profesorado se sienta más identificado con la misión de la institución, puesto que puede tomar decisiones que antes posiblemente no podría por miedo a equivocarse o no estar en la misma sintonía que el resto del profesorado o que el propio colegio.

Si nos centramos en las familias, estas pueden acceder a la información relacionada con sus hijos e hijas, que se actualiza en tiempo real y que les va siendo facilitada tanto por parte del profesorado, como por el departamento de orientación y la dirección. Además, ven al colegio con una imagen de modernidad que está en constante actualización, adaptándose al mundo tecnológico actual, que apuesta por el uso eficiente de la información y transparencia en la gestión de la información, y sin coste económico para las familias.

Lo importante y trascendente de este proyecto es que es reproducible en cualquier centro educativo, al ser muy fácil su puesta en marcha por no requerir conocimientos de programación (excepto para el desarrollo de scripts a medida y otras personalizaciones, como la comunicación personalizada en el aviso de las faltas de asistencia y/o retrasos, e incidencias y/o felicitaciones). 
Si el equipo directivo lo impulsa, cualquier profesor/a con iniciativa y ganas de mejorar la comunicación en su centro puede crear la estructura de la plataforma que luego dotará de contenidos conforme se vayan creando y vayan siendo necesarios en su centro. Si la persona responsable de elegir la información tiene capacidad de decisión, por formar parte del equipo directivo o haber sido autorizada por este, mucho mejor, puesto que tendría capacidad de decisión para determinar qué información puede o no puede publicar sin necesidad de pedir autorización para publicarla. En la puesta en marcha de este proyecto también hay un beneficio ecológico: se ahorra gran cantidad de papel y energía puesto que no es necesario imprimir la información que antes se tenía que copiar y repartir tanto al profesorado como a las familias. La información está actualizada en tiempo real. Se ahorra tiempo a las familias, al poder recibir la información de sus hijos e hijas por la plataforma, no siendo necesario tener que desplazarse al colegio ni realizar llamadas telefónicas para ponerse en contacto con el profesorado y recibir la información deseada. También se observa una disminución de la información no formal y de los rumores por parte de las familias, lo que fomenta un rigor en la información que trasciende del colegio. La imagen y el trabajo corporativo colaborativo mejora, ya que al disponer todos de la misma información y las herramientas necesarias para procesarla aumenta la coordinación y, por lo tanto, la eficiencia por un mejor aprovechamiento del tiempo y, sobre todo, de las experiencias anteriores.

\section{BiBLIOGRAFÍA}

Boersma, K. y Sytze, K. (2011). Organizational Learning Facilitation with Intranet (2.0), a Socio-Cultural Approach, en Organizatonial Learning and Knowledge (cap. 517).

Casares, E. (2007). La comunicación en la Organización; Retroalimentación como fuente de satisfacción. Razón y Palabra. Recuperado de: http://www. cem.itesm.mx/dacs/publicaciones/logos/anteriores/n56/ecasares.html.

Comern (2014). Revista de los estudios de ciencias de la información y de la comunicación. Recuperado de: http://www.uoc.edu/divulgacio/comein/es/ numero36/articles/Article-Josep-Cobarsi.html.

FERnÁNDEZ, C. (1997). La comunicación en las organizaciones. Trillas. 
GonzÁlez, M. (2015). Habilitando una organización que aprende e innova mediante la Intranet. Ciudad de México: Universidad Iberoamericana.

Katz, D. y Kahn, R. (1990). Psicología Social de las Organizaciones. México: Trillas.

Latorre, A. (2003). La investigación acción. Conocer y cambiar la práctica educativa. Grao.

Martínez de Velasco, A., Nosnik, A., Vargas, V. y Savage, G. (1988). Comunicación horizontal, informal y rumor. México: Trillas.

MECD (2015, sept.).Educ@conTIC. Recuperado de: http://www.educacontic.es/ blog/usando-google-apps-para-educacion (consulta: 1/6/2016).

Nosnik, A. (1990). Las tres "ies” de la comunicación organizacional en Alta Dirección.

Nosnik, A. (1996). Comunicación y gestión organizacional. Bucaramanga, Colombia: Universidad Autónoma de Bucaramanga.

Santana, C. (2013). Clima Organizacional, Productividad, Recursos Humanos. Recuperado de: http://www.acsendo.com/es/blog/la-importancia-dela-comunicacion-interna-en-las-organizaciones/.

SoRIA, R. (2008). Comunicación organizacional: un modelo aplicable a la microempresa. Teacs, 9-25.

Purísima-Franciscanas, C. L. (2015, septiembre). RRI. Reglamento de Régimen Interior. Valencia. 